\title{
Sensory equipment and adaptations to the fire habitat of the antennae of the Australian 'firebeetle' Merimna atrata (Coleoptera; Buprestidae)
}

\author{
Anke Schmitz ${ }^{1} \cdot$ Helmut Schmitz ${ }^{1}$ (D)
}

Received: 16 June 2021 / Revised: 4 October 2021 / Accepted: 12 October 2021 / Published online: 20 October 2021

(c) The Author(s) 2021

\begin{abstract}
The 'Australian firebeetle' Merimna atrata approaches fires in Eucalyptus forests for reproduction. Beetles stay on a postfire area as long as burning wood or hot ashes emit heat and smoke. Abdominal infrared receptors protect the beetles from landing on hot spots; however, until now fire-specific adaptations of the antennae have not been investigated in more detail. This affects the localization of olfactory sensilla used for the perception of smoke and in addition mechanisms to protect delicate sensilla against desiccation and pollution. Moreover, nothing was known about antennal thermo-/hygroreceptors in Merimna atrata. We found strong evidence for a functional grouping of the sensilla into receptors used on the ground or in flight, respectively. A first group comprises the outer visible sensilla, i.e. mechanosensory bristles, short gustatory sensilla and a small field of very short olfactory sensilla. They are used when the beetle is running around on the fireground on burnt bark or ashes. A second group of sensilla is hidden in closeable cavities on antennomeres 4-11. If the cavities are closed, the sensilla inside are fully protected. If the cavities are opened in flight, the beetles can make use of many multiporous basiconic sensilla and multiporous basiconic grooved peg sensilla for smoke detection. Minute modified sensilla coelocapitula occurring in small numbers in the cavities too, most probably serve as thermoreceptors. As a result the placing of sensilla deserving protection in closeable cavities and the reduction in number and length of the external sensilla can be interpreted as adaptations to the fire habitat.
\end{abstract}

Keywords Jewel beetles $\cdot$ Pyrophilous insect $\cdot$ Antennal sensilla $\cdot$ Thermoreceptor $\cdot$ Sensillum coelocapitulum

\section{Introduction}

The 'firebeetle' Merimna atrata represents the only species within its genus and is endemic to Australia and the southern part of New Guinea (Bellamy 2002; Hawkeswood 2007). Moreover, this deep black jewel beetle is strongly attracted by forest fires (Tepper 1887; Poulton 1915). According to the current knowledge, adaptations to fires are so extensive that the survival of a population of Merimna in an area devoid of periodic fires in Eucalyptus forests is not possible (Schmitz et al. 2015; Schmitz and Schmitz 2020). Males and females likewise approach ongoing forest fires and the fireground serves as a meeting place for the sexes (Schmitz

Helmut Schmitz

h.schmitz@uni-bonn.de

1 Institute for Zoology, University of Bonn, Meckenheimer Allee 169, 53115 Bonn, Germany and Schmitz 2002). Copulations can already be observed a few hours after the blaze and after mating the females deposit their eggs under the bark of the freshly burnt trees. After 2-3 days, when all pockets of embers have cooled down, most beetles already have left the burnt area (Schmitz et al. 2015).

The wood of the trees severely injured or killed by fire is a valuable source of food for various kinds of xylophagous insects like most buprestid larvae. The distinctive capability of Merimna to detect fires in Eucalyptus forests ensures that this pyrophilous beetle can start reproduction when smoke and heat still keep away competitors. Additionally, predators like birds are also kept away. On the other hand, sojourning on a freshly burnt area bears a high risk. Due to high temperatures, low humidity and many hot surfaces the "active" fireground during the first two postfire days is an extreme and, therefore, rather dangerous place not only for insects (Schmitz et al. 2015). Special sensory adaptations are necessary for coping with the hazardous conditions. To 
avoid a landing on a hot surface Merimna is equipped with unique abdominal infrared (IR) receptors (Schmitz et al. 2000). However, another sensory organ most probably used for fire detection as well as for orientation and survival on burnt areas, the antenna has not been investigated in much detail. Therefore, the possible fire adaptations including special sensors of this exposed but fragile structure are in the focus of interest in our study.

Although the jewel beetles (Buprestidae) are a large beetle family that contains also economically significant species, publications about the structure and morphology of antennal sensilla are relatively rare (Scott and Gara 1975; Volkovitsh 2001; Crook et al. 2008; Yi et al. 2016; Bari et al. 2019; Faucheux et al. 2020). In most papers, the external morphology of the sensilla is described by SEM; only in one paper additional TEM micrographs are presented revealing the innervation pattern (Crook et al. 2008). The gross morphology of the Merimna antenna has been briefly described in the comprehensive work of Volkovitsh dealing with the morphology of antennal structures of more than 300 buprestid genera (Volkovitsh 2001). However, results have not been discussed in detail with regard to the pyrophilous biology of Merimna. Two peculiarities were mentioned that could be of importance for the pyrophilous behaviour of Merimna. First, a so-called apical organ on most of the 11 antennomers has been described. Apical organs, also termed slit organs, are cavities at the anterior margins of antennomer 4-10 and at the top of the terminal 11th antennomer. Inside the cavities that can be completely closed, many sensilla are located. The sheltered location of these small sensilla may be a protective mechanism. Secondly it was described that most sensilla are very short, possibly interpretable as an adaptation to dry and dusty conditions (Volkovitsh 2001).

Apical organs have already been described in 1975 by Scott and Gara in another pyrophilous jewel beetle, the "charcoal" beetle Melanophila acuminata. The genus Melanophila is closely related to Merimna and shows a nearly identical pyrophilous behaviour in the Palaearctic region. Scott and Gara suggested that antennal sensilla in Melanophila may play a role in short-range orientation on the fire ground preventing beetles from coming too close to hot surfaces. Therefore, the main goal of the study was the detection of antennal thermoreceptors and the possible existence of olfactory receptors used for host selection. The authors showed that the apical organs contain 15 to more than 60 sensilla basiconica and found morphological evidence that these sensilla act as chemoreceptors. However, the details of the inner structure of the apical organs are still unknown. The authors also speculated that the apical organs can be actively opened and closed to concentrate odour molecules inside the cavities of the slits. Additionally, they describe "campaniform-like organs" on the lateral regions of the female antennomers that may work as thermoreceptors.
However, no further evidence is provided to corroborate this statement.

Consequently, a multitude of specific adaptations of the antenna of Merimna can be proposed like an increased abundance of thermo-/hygroreceptors, numerous olfactory receptors for the detection of smoke and structural adaptations to protect the delicate sensilla against desiccation and contamination. Consequently, the intention of our investigation is the ultrastructural characterization of all antennal sensilla allowing a prediction about their possible function and the description of possible fire-specific features of the antenna as a whole.

\section{Material and methods}

\section{Animals}

Merimna atrata (Gory and Laporte 1837) were caught in Western Australia in January 2020 on burnt areas after forest fires in the Perth metropolitan region (Licence No.: FO25000232, Department of Biodiversity, Conservation and Attractions, WA). Beetles were kept in small plastic boxes for a few days and fed with peanuts, almonds and raisins. Antennae were removed and processed in a laboratory at the WA Wildlife Research Centre in Woodvale. The experiments complied with the Principles of Animal Care of the National Institute of Health and with the laws of Germany (Tierschutzgesetz).

\section{Scanning electron microscopy}

Isolated antennae were fixed in $70 \%$ ethanol and cleaned by several rounds of sonication (3-4 min. each), first in fresh $70 \%$ ethanol and subsequently in a 1:2 mixture of ethanol/ chloroform. After a brief wash in $70 \%$ ethanol, the specimens were dipped in a small dish with lens cleaner $(\mathrm{B}+\mathrm{W}$ Filter) and air-dried. Dried antennae were mounted on stubs with double-sided adhesive Leit-Tabs (Plano GmbH, Wetzlar, Germany) sputtered with gold and investigated in a Cambridge SEM.

\section{Light and transmission electron microscopy}

Freshly excised antennae were immediately transferred into iced glutaraldehyde fixative (3\% glutaraldehyde in $0.05 \mathrm{~mol} \mathrm{l}^{-1}$ cacodylate buffer, $\mathrm{pH} 7.1,400 \mathrm{mosmol}^{-1}$ ). To allow the fixative access to the tissue, the antenna were cut into pieces of three to four antennomers. Antennomers earmarked for cutting were carefully laterally opened or incised by a razor blade splinter clamped in a holder or pierced with a fine needle. The remaining antennomers served for handling the fragment. Specimens were then fixed overnight, 
subsequently washed in buffer and postfixed with $1.5 \%$ $\mathrm{OsO}_{4}$ in the same buffer. Afterwards, specimens were dehydrated through an ascending ethanol series and embedded in Epon 812 using propylene oxide as a bridging solvent. Semithin and ultrathin sections were cut with a Reichert Ultracut Microtome using glass or diamond knives. Semithin sections with a thickness of $0.5 \mu \mathrm{m}$ were stained with a $0.05 \%$ toluidine-blue/borax solution and examined with a Zeiss Axioscope 5 light microscope. Identification of the different cuticular types was achieved by Mallory's trichrome stain adapted to insect tissue (Weyda 1982). According to the staining protocol, the endocuticle appears blue, the mesocuticle red and the (hard) exocuticle remains more or less unstained. Photos were taken with a Jenoptik Gryphax Arktur. Ultrathin sections were stained with uranyl acetate and lead citrate and examined with a Zeiss Libra 120 TEM at $60 \mathrm{kV}$.

Sections were taken from several antennomers of two males and two females.

\section{Measuring of sensilla}

Adobe Photoshop CC 2021 was used to measure the dimensions of the different sensilla. Because more than $50 \%$ of the sensilla are hidden in cavities, the quantification of the numbers of the visible sensilla only appears of limited use for the characterization of the antennal sensory system and, therefore, was not carried out. Moreover, the sensilla on the outside are arranged in low density and, therefore, are easy to identify (cf. Fig. 1). Thus, a semiquantitative assessment of their numbers is provided.

\section{Results}

\section{General morphology of the antenna}

As described in the paper of Volkovitsh (2001), the antenna of Merimna consists of 11 antennomers: scape and pedicel and nine flagellar segments. Except for the basal three antennomers, the distal eight antennomers are bilaterally flattened and exhibit a serrate-truncate appearance (Fig. 1A). The outermost distal antennomer 11 has a rounded tip (Figs. 1A and 2C). Because of the large differences in size of the beetles (body length from $1,5 \mathrm{~cm}$ to $3.5 \mathrm{~cm}$ ), the length of the antennae also differs between 4 and $7 \mathrm{~mm}$.

Distally, antennomers 4-11 bear sensory cavities. These cavities are discernible by a semicircular slit around the anterior truncated edge of the antennomer (Figs. $1 \mathrm{~A}-\mathrm{C}$ and 2C). In dried antennae, the slits usually are closed so that the sensilla inside the cavities are not visible. A view onto the ventral side revealed that the arcuate slit is formed asymmetrically (Fig. 1C). In the forward facing antennae of active beetles, the slits on the outsides of the antennomers are much longer than on the insides (see arrowheads in Fig. 1C). In flight, the antennae point outwards in an angle of about $20^{\circ}$ related to the longitudinal axis of the beetle (Hinz et al. 2018). If a cavity is slightly opened, the incoming air flows into the short inner part of the slit (see arrow in Fig. 1C).

On the antennomers, the sensilla are arranged quite irregularly. Most external sensilla are located on the ventral half of an antennomer. In general, five fields of sensilla can be distinguished (cf. Fig. 1B): (1) on the anterior edge of the dorsal surface of antennomers 4-11, a small group of basiconic gustatory sensilla $\left(G_{\text {dorsal }}\right)$ is located. Sensilla are more or less arranged in a row transverse to the longitudinal axis (Figs. 1B and 2C). (2) The remaining dorsal half as most of the lateral regions are sparsely covered by trichoid mechanosensory bristles (M). (3) The sensory cavities (C) are discernible by the mentioned slit and contain basiconic, coelocapitular and grooved peg sensilla. (4) A broad field of basiconic gustatory sensilla $\left(\mathrm{G}_{\mathrm{ventral}}\right)$ covers most of the ventral bulge of antennomers 4-11. A few tiny mechanosensitive bristles are also present in this field (Figs. 1B, C and 2A, C). (5) Basiconic olfactory sensilla (O) cover the posterior third of the ventral bulge of antennomers 4-11 (Figs. 1B, C and 2A).

In the following, the sensilla of the different fields and inside the cavities will be characterized with regard to their morphology and possible function.

\section{Mechanoreceptors of the dorsal and lateral fields}

Mechanosensitive bristles (sensilla trichodea) cover all antennomers. However, the density of mechanosensitive bristles is low (Fig. 1A, B) and the bases of the bristles are innervated by a single mechanosensitive receptor cell characterized by a well-developed tubular body (Fig. 2D). Consequently, the bristle lumen does not contain any dendrites. Furthermore, the distribution of s. trichodea is heterogeneous. As repeated examinations of scapus and pedicellus showed, only sensilla trichodea were found here. The following antennomer 3 also mainly bears s. trichodea. However, anteriorly a few basiconic gustatory sensilla (see below) can already be found. On all following antennomers 4-11 showing sensory cavities, the anterior third of the dorsal surface as well as the area around the cavities is devoid of s. trichodea (Figs. 1B and 2C). In anterior ventral direction, bristles become considerably shorter (Figs. 1B and 2A). Whereas bristles of dorsally located s. trichodea are up to $50 \mu \mathrm{m}$ long, the bristles of anterior ventrally located sensilla are only about $5 \mu \mathrm{m}$ long (Figs. 1B and $2 \mathrm{~A}-\mathrm{C}$ ). As a special feature, some of the short bristles show two tiny tips (Fig. 2B). As a rule, the short bristles are interspersed between the gustatory s. basiconica and, therefore, do not overtop the contact gustatory receptors (Fig. 2A-C). The same tendency 


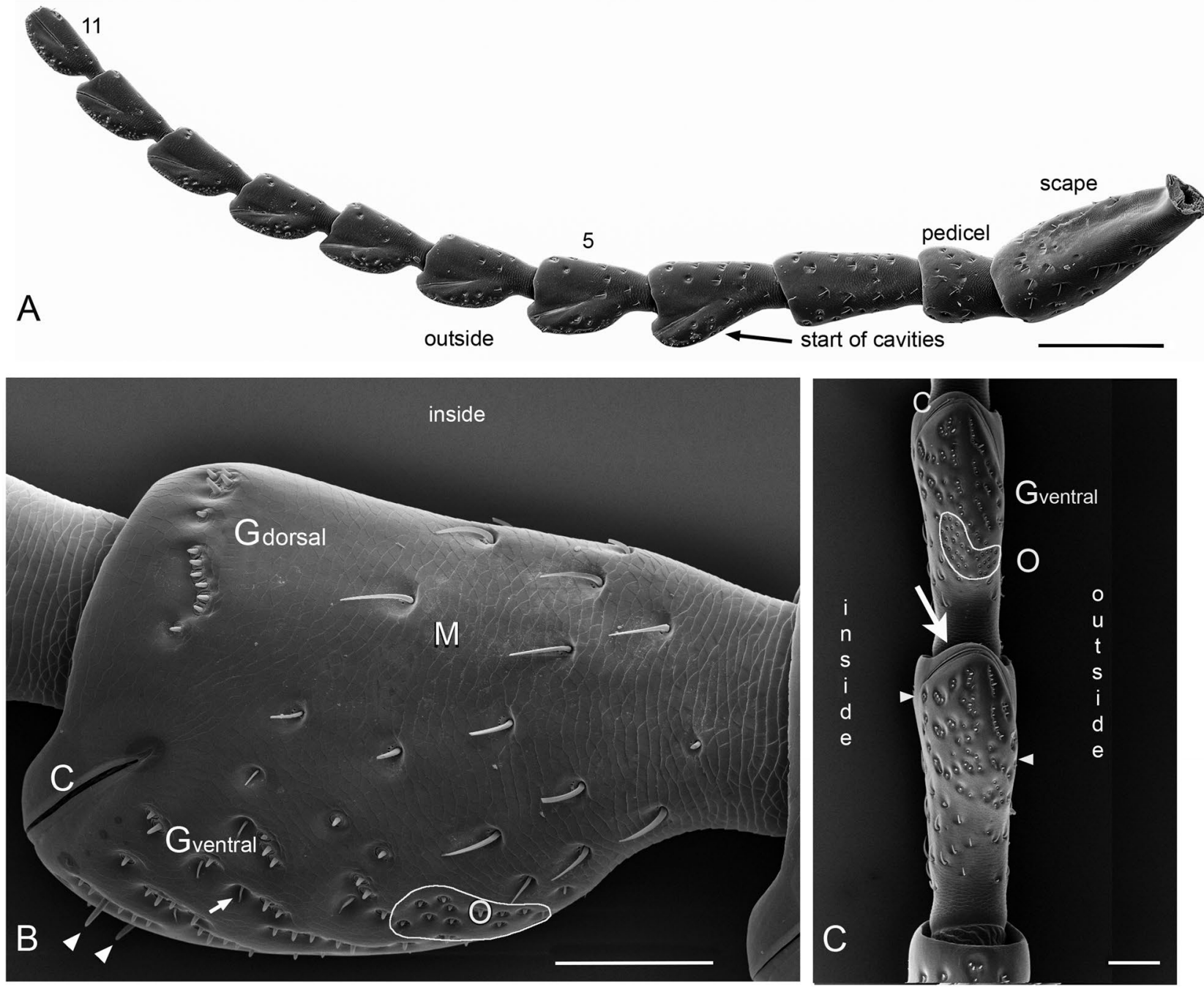

Fig. 1 Outer morphology of the antenna. A Left antenna of a male cut at the base of the scape. The truncate/serrate antenna consists of 11 antennomers and has a total length of $5 \mathrm{~mm}$. Cavities are situated on antennomers 4-11. On the outside, the slit-shaped openings of the cavities extend almost to the middle of the respective antennomers; bar: $500 \mu \mathrm{m}$. B Inner face of antennomer No. 5. Five fields of sensilla are clearly visible: namely the anterior dorsal field of gustatory sensilla $\left(\mathrm{G}_{\mathrm{dorsal}}\right)$, the posterior field of mechanosensory bristles $(\mathrm{M})$, the posterior ventral field of olfactory sensilla $(\mathrm{O}$, encircled), the cavity $(C)$ and the anterior ventral field of gustatory sensilla $\left(\mathrm{G}_{\mathrm{ventral}}\right)$. Arrowheads indicate two longer gustatory sensilla and small arrow points to a single small mechanosensitive bristle within this field; bar $100 \mu \mathrm{m}$. C Ventral view of antennomers 4 and 5. Arrowheads indicate beginnings of the slit openings on the inside and outside. Labelling of the sensillar fields as in B. Arrow indicates direction of airflow in flight; bar $100 \mu \mathrm{m}$ of reduction in anterior ventral direction can be observed in the accompanying wax glands. Long dorsal s. trichodea (large arrow in Fig. 2A) have several pores of wax glands incorporated in their socket region (see inset in Fig. 2A): a feature very common in jewel beetles (see Discussion). No such openings can be found in the short s. trichodea located ventrally (Fig. 2A-C).

\section{Gustatory sensilla of the dorsal and ventral fields}

The basiconic sensilla located in the dorsal and ventral fields $\left(\mathrm{G}_{\mathrm{dorsal}}\right.$ and $\left.\mathrm{G}_{\text {ventral }}\right)$ shown in Figs. 1B, C and 3A belong to the same type and, therefore, are dealt with together in this chapter. Most sensilla have a short peg with a length of about $6 \mu \mathrm{m}$ showing one or two characteristic notches in their wall (arrowheads in Fig. 3A, C). Sensilla are frequently arranged in rows (cf. Figs. 1C, D and 3A). In both the dorsal and the ventral fields, a few sensilla can be found that show significantly longer pegs (three to four times as long as the common sensilla, arrow in Fig. 3A). If a sensillum with a longer peg is integrated into a row, it is usually situated in the middle (as shown in Fig. 3A). Pegs are based in a large socket membrane that-in dried antennae-show a wrinkled surface. Sections through the peg revealed a pore at 
Fig. 2 Mechanoreceptors. A View onto the ventrolateral inner surface of a male antenna (7th antennomer). Large arrow points to a sensillum trichodeum with a long bristle and a number of wax pore openings in the socket region. Inset shows pores at higher magnification (bar: $5 \mu \mathrm{m}$ ). Anteriorventrally, s. trichodea gradually become smaller and openings of wax pores are no longer present. Arrow points to a small anterior s. trichodeum. Arrowheads label two longer gustatory sensilla basiconica; O: small field of olfactory s. basiconica; bar: $70 \mu \mathrm{m}$ : B Small s. trichodeum between gustatory s. basiconica in the anteroventral region of the antennomer. Note the two tips of the bristle; bar: $10 \mu \mathrm{m}$. C Tip of the 11th antennomer showing rows of gustatory s. basiconica interspersed with small s. trichodea; ventral side on the left; bar: $50 \mu \mathrm{m}$ D Oblique section through the bristle base of a small s. trichodeum. The outermost tip of the dendrite with the tubular body (TB) is embedded in the socket septum (SS) and in the cuticle $(\mathrm{C})$ of the base of bristle; bar: $0.5 \mu \mathrm{m}$
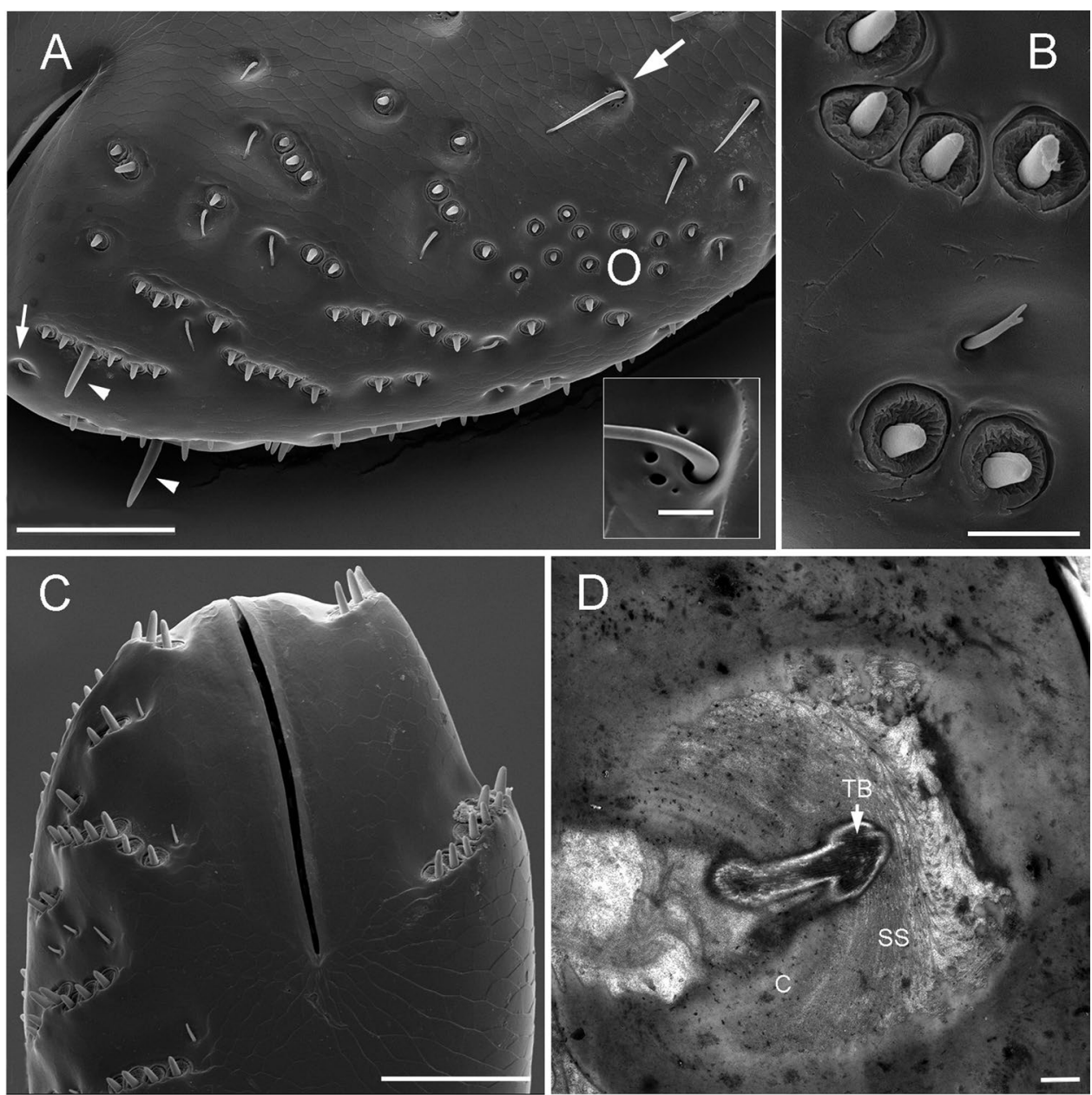

its outermost tip (Fig. 3B). However, no lateral pores were found in the wall. This is consistent with the finding that the dendrites extending into the peg are surrounded by a dendritic sheath up to the tip (Fig. 3B, C). By this arrangement, typical for gustatory sensilla, a dendritic-free canal is separated from the lumen containing the dendrites (Fig. 3B-D). As already described in the pioneer work of Gaffal, the dendritic-free canal represents an apical prolongation of the outer sensillum lymph space (Gaffal 1979). The pore is occluded by electron-dense material (arrow in Fig. 3B).

Cross sections through a region below the base of the peg at the level of the socket membrane reveal dendritic outer segments (DOS) of several receptor cells (Fig. 3D, E). One DOS ends up here and contains a tubular body characteristic of a ciliary mechanoreceptive receptor cell (Fig. 3E). In addition to the mechanoreceptor, five to six cylindrical DOS of receptor cells extend into the lumen of the peg. We found no evidence that the dendrites branch inside the peg.

Every gustatory sensillum basiconicum is accompanied by a wax gland (Figs. 4 and 5A). Distally, the wax gland ends up right next to the socket membrane below a region of extremely thin cuticle (about $0.6 \mu \mathrm{m}$, Figs. $3 \mathrm{~F}$ and $4 \mathrm{~A}$ ). In this area, numerous micropores penetrate the cuticle (arrowheads in Fig. 3F, pores not well visible). Obviously, the micropores serve for releasing the wax. Below the thinned cuticle, a cuticular tube containing the large wax gland extends down to the haemocoel (Fig. 4B-C). In Figs. 4D and $5 \mathrm{~A}$, it can be seen that the proximal part of the wax gland is in direct contact to the haemolymph by an elaborate basal labyrinth.

\section{Olfactory sensilla of the posterior ventral field}

The small field of multiporous sensilla with short pegs (Figs. 1B, C and 6A) is located ventrally on antennomers 4-12. Sensilla are situated on the posterior part of the ventral bulge of the antennomers; the most proximal sensilla can be found on the posterior curvature of the bulge. Initially, sensilla appear in small numbers of less than ten on the four antennomer. From antennomer 5 on, the number gradually increases and, on the distal antennomers, up to 30 sensilla can be found. However, on the 11 . antennomer, the number decreases again to less than 20 . There is no sharp border between the fields of gustatory 
Fig. 3 Gustatory sensilla of the ventral and dorsal fields. A Basiconic gustatory sensilla of the ventral field (cf. Fig. 1B, C). Anteriorly, the linearly arranged groups of sensilla often contain a central sensillum with a longer peg. Sensilla with long pegs (arrow) are also interspersed between rows of sensilla with short pegs. Arrowhead points to notches in a peg; bar: $10 \mu \mathrm{m}$. B Longitudinal section through the tip. The tip pore (arrow) is occluded by an electrondense plug; $F C$ dendritic-free canal, $D S$ dendritic sheath; bar: $0.5 \mu \mathrm{m}$. C Cross section through the distal part of a peg. The massive cuticular wall shows no pores. In the inner lumen 5 dendrites are surrounded by a dendritic sheath (DS). The dendritic-free canal (FC) surrounds the DS. Note notch in the wall of the peg (arrowhead); bar: $0.5 \mu \mathrm{m}$. D Cross section through the base of the peg. The dendrites are enclosed in a common dendritic canal separated from the additional dendritic-free canal (FC); bar: $1 \mu \mathrm{m}$. E Cross section a few $\mu \mathrm{m}$ below the peg base. Six dendrites and the tubular body (TB) of the mechanosensory cell can been seen; bar: $0.5 \mu \mathrm{m}$. F The dendritic canal (on the right, asterisk) is accompanied by a large wax gland (WG) encased in a cuticular tube. The wax is released through micropores (arrowheads) crossing a thin cuticle next to the socket region of the sensillum; bar: $3 \mu \mathrm{m}$
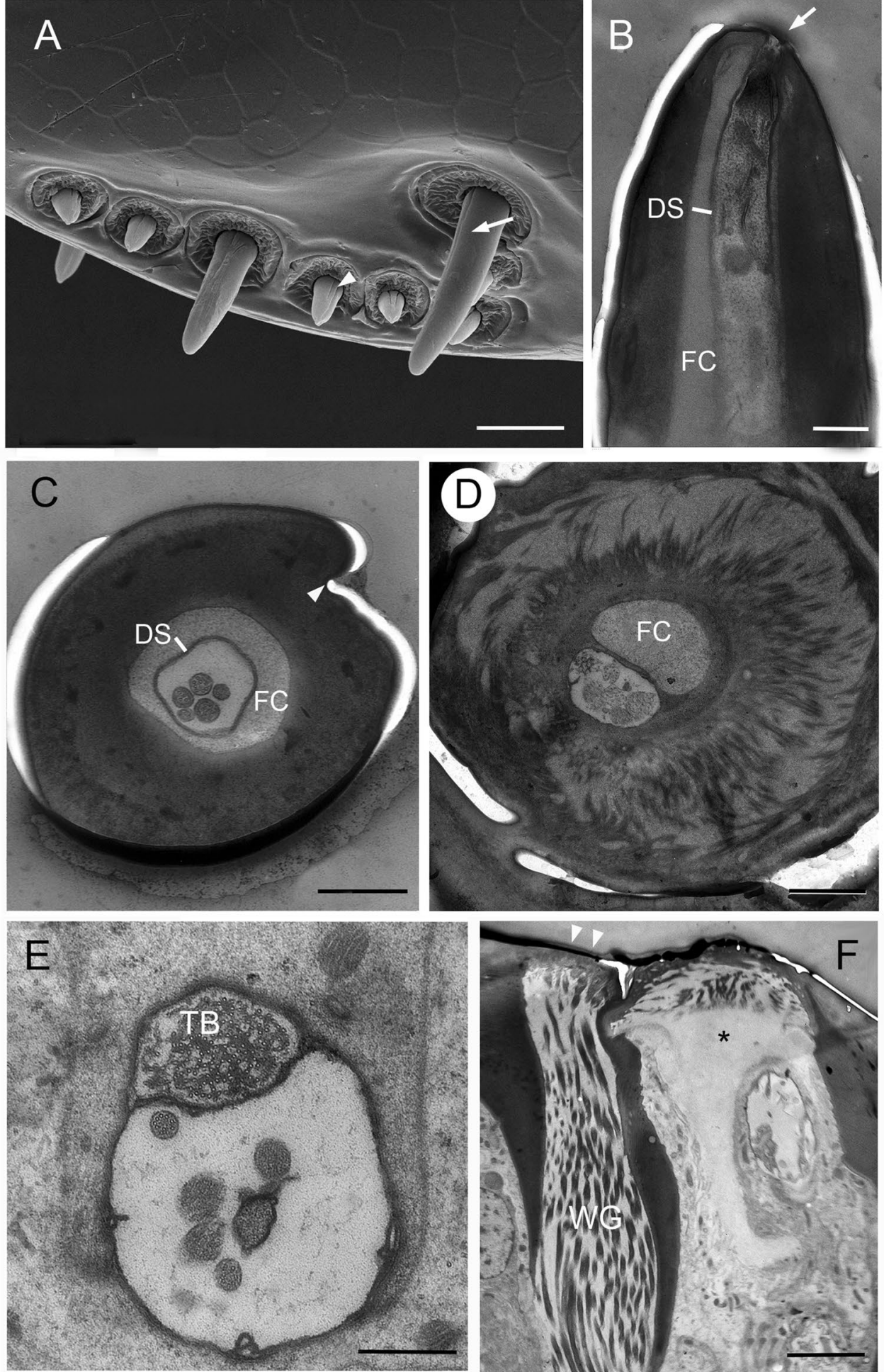

and olfactory sensilla. However, in contrast to the linearly arranged gustatory sensilla, the olfactory sensilla occur isolated and are slightly shorter (length of the peg 3-4 $\mu \mathrm{m})$. Like the gustatory sensilla described above, the olfactory sensilla are also accompanied by a wax gland. Fig. 6A shows the micropores next to the socket (arrow).
Because no auxiliary cell forming the conducting canal and the pore opening was found, the wax gland is unicellular and, therefore, can be classified as class-1 gland cell (Noirot and Quennedey 1991). In Fig. 6G, H the wax gland (WG) partly surrounds the dendritic canal inside the cuticle. 
Fig. 4 Wax glands I. A Gustatory s. basiconicum with associated wax gland (WG) that is enclosed by a cuticular tube. Note thin exocuticle penetrated by numerous micropores (arrow); bar: $10 \mu \mathrm{m}$. B Detail of a wax gland (WG) next to a s. basiconicum (asterisk). Most of the enveloping tube is visible; bar: $10 \mu \mathrm{m}$. C Basal end of the cuticular tube opens to the haemocoel (arrow); bar: $10 \mu \mathrm{m}$. D Basal orifice of the canal. The proximal part of the gland cell is exposed to the haemolymph. A distinct basal labyrinth (arrow) facilitates nutrient uptake, bar: $10 \mu \mathrm{m}$
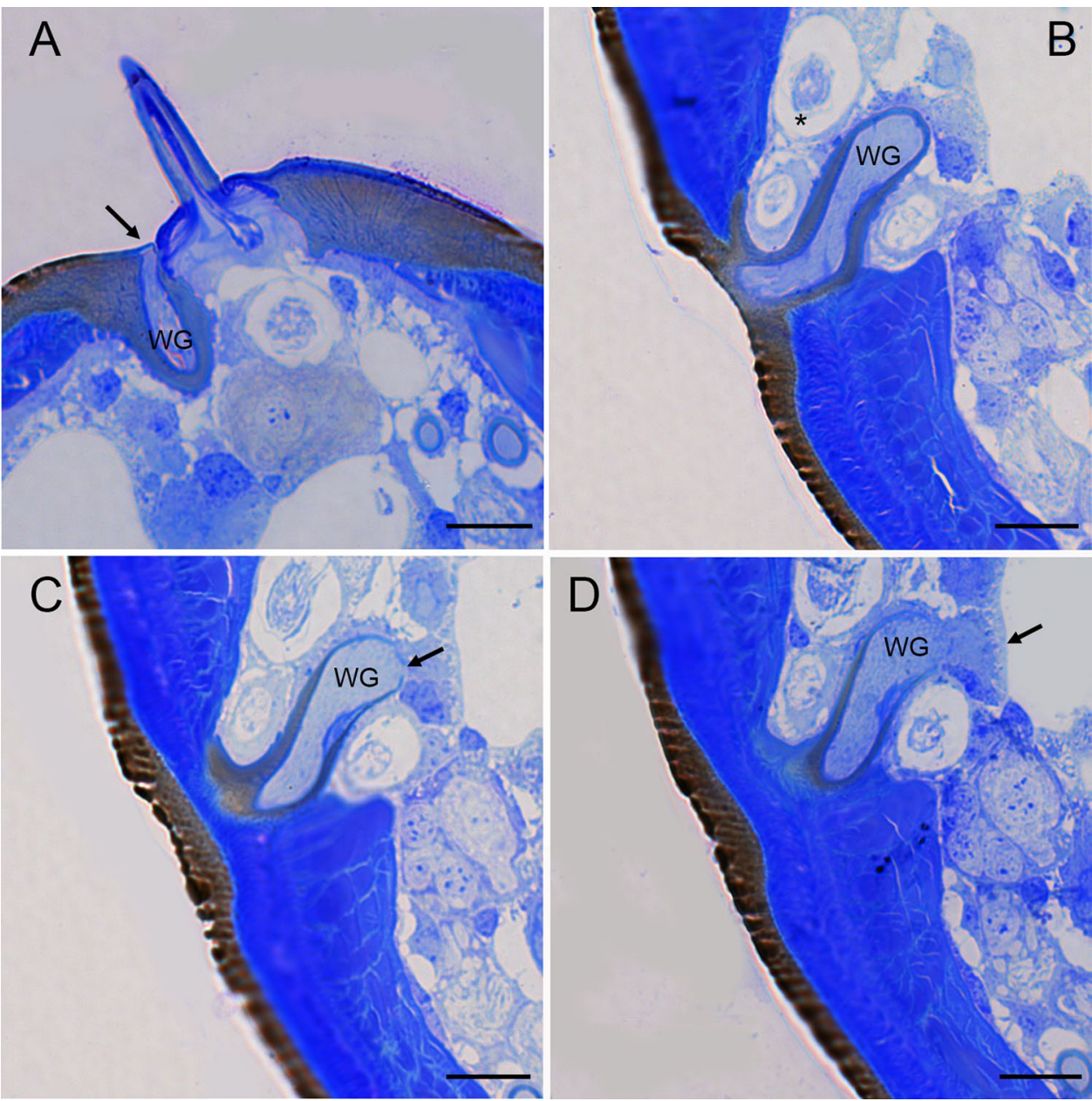

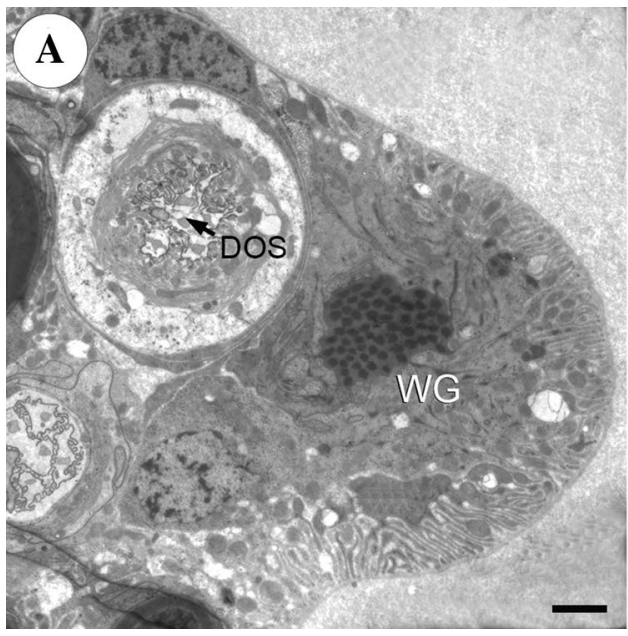

Fig. 5 Wax glands II. A. Cross section through a gustatory sensillum basiconicum (DOS: dendritic outer segments of sensory cells inside receptor lymph cavity) and the adjacent wax gland (WG, unicellular class-I cell). Plane of the section proximal to the cuticular tube. The dark wax strands inside the lumen of the gland are well visible. Note

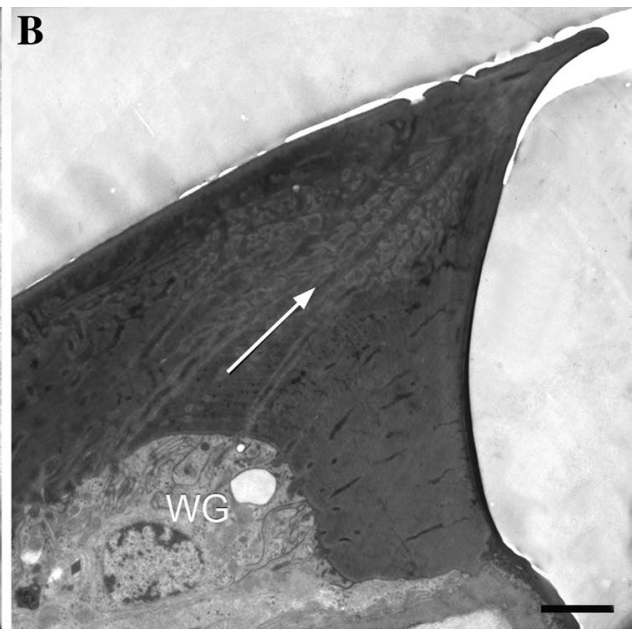

basal labyrinth exposed to the haemolymph; bar: $2 \mu \mathrm{m}$. B. Partial view of the distal region of a cavity. Arrow indicates direction of wax canals originating from a wax gland (WG) and running straight to the outer lip of the cavity; bar: $2 \mu \mathrm{m}$ 

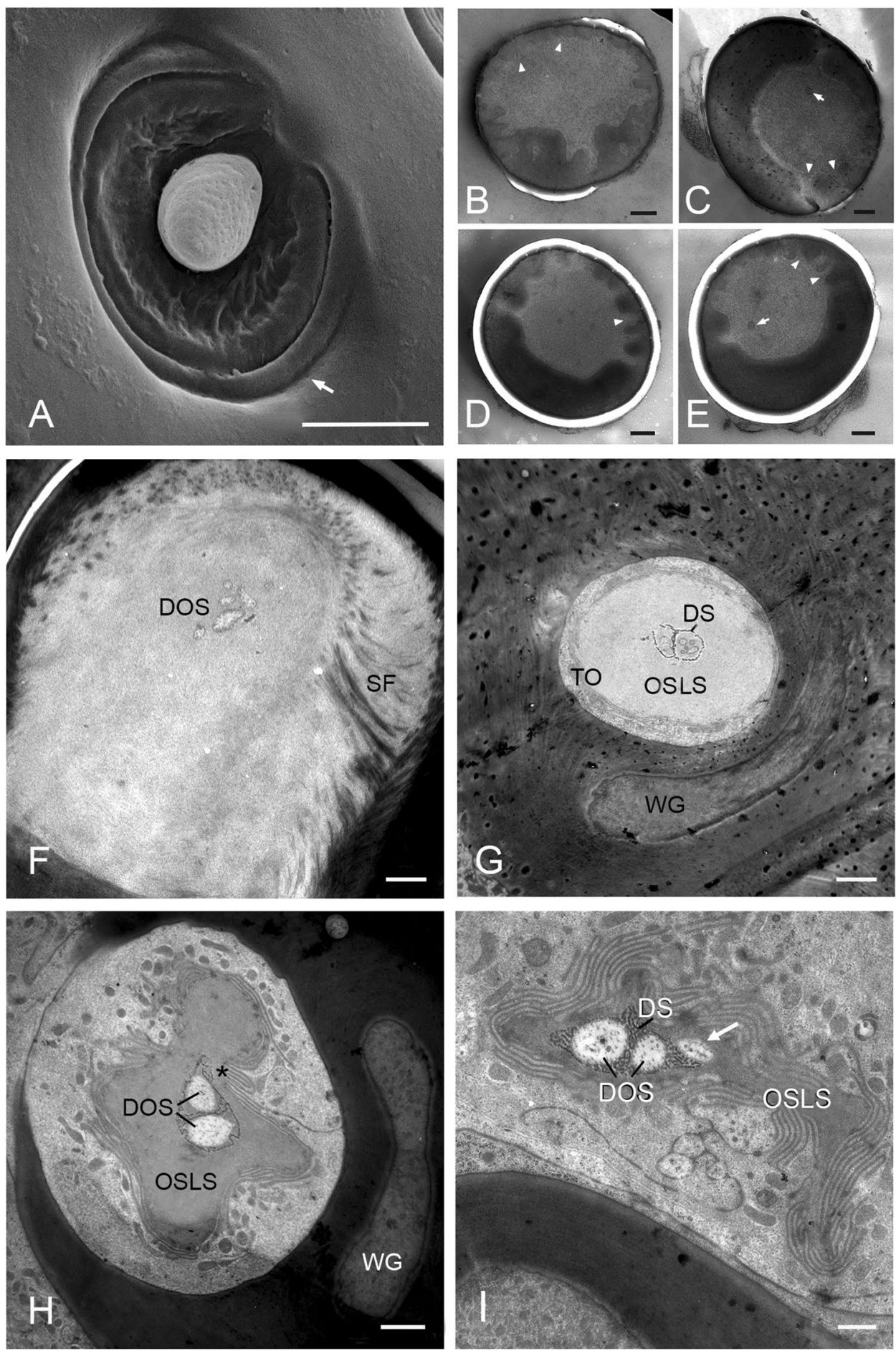

Sensilla are single-walled and cross sections through pegs show numerous pores with pore tubuli (Fig. 6B-E, arrowheads). Although we investigated sections through pegs of many sensilla, a noteworthy number of dendrites could never be identified inside the lumina. Thus, the number of thin terminal dendrites with diameters of about $70 \mathrm{~nm}$ seems rather small (Fig. 6C, E, small arrows). A low number of dendrites inside the peg is confirmed by a cross section through the base of the peg (Fig. 6F) and the outer sensillum lymph space (OSLS) located directly below the cuticle and also extending into the cuticular canal (Fig. 6G) where branching of dendrites starts. Only few dendritic branches are visible. A section through the OSLS just below the cuticular canal shows two unbranched dendritic outer segments (DOS), suggesting that two receptor cells innervate each sensillum (Fig. 6H). However, in one sensillum, we found a presumed 
४Fig. 6 Olfactory sensilla of the posterior ventral field. A SEM micrograph of a single sensillum. Note numerous pores in the wall of the peg. A wrinkled joint membrane surrounds the peg. An incomplete circular cleft separates the joint membrane from the slender socket. The accompanying wax gland opens into micropores (arrowheads) located on the socket and the adjacent cuticle; bar: $3 \mu \mathrm{m}$. B-E Cross sections through the pegs of four different sensilla. Wall pores and pore tubuli (arrowheads) are visible. Terminal dendritic branches are rare and are clearly visible only in $\mathrm{C}$ and $\mathrm{E}$ (short arrows). Oblique sections through the basal regions of the pegs $(\mathbf{C}-\mathbf{E})$ show massive regions of the walls without pores; bars: $0.25 \mu \mathrm{m}$. F Cross section through the apical end of the dendritic canal. In the upper corner, the socket septum with included suspension fibres (SF) is visible. Only a few dendritic outer segments (DOS) further extending into the lumen of the peg can be discerned; bar: $0.5 \mu \mathrm{m}$. G Section through the dendritic canal in the cuticle. The tormogen cell (TO) exhibiting microvilli encompasses a large outer sensillum lymph space (OSLS). Two dendritic outer segments surrounded by partly distorted dendritic sheaths (DS) are located in the middle of the cavity; the right dendrite has already branched in 5 smaller dendrites. A large wax gland (WG) encloses about one third of the canal; bar: $1 \mu \mathrm{m}$. H Section through the outer sensillum lymph space (OSLS) at the proximal opening of the dendritic canal below the socket septum. The distal enveloping cell (most probably the tormogen cell) forms numerous microvilli. Two dendritic outer segments (DOS) encircled by slightly deformed dendritic sheaths are visible. Note accompanying wax gland (WG); bar: $1 \mu \mathrm{m}$. I Section through the basal region of the outer sensillum lymph space (OSLS). The tormogen and the trichogen cell form an extensive labyrinth of microvilli. Two dendritic outer segments (DOS) encircled by fractionated dendritic sheaths (DS) are visible. A third putative DOS (arrow) not enclosed by a dendritic sheath may be present; bar: $0.5 \mu \mathrm{m}$

DOS of a third receptor cell in the basal region of the OSLS below the cuticle (Fig. 6I). Nevertheless, we found no evidence that a third DOS extends with its dendrites into the peg lumen. Furthermore, no evidence for a mechanosensory innervation of the peg (i.e. a tubular body) could be found.

\section{Design and morphology of the cavities and the internal sensilla}

As described above, cavities are located on antennomers 4-11 (cf. Fig. 1A). In most of the dried beetles investigated, the cavities are closed; however, most probably, depending on the drying process, in some specimens the cavities are slightly opened (Fig. 1B). This shows that the cavities can be in an open or closed state. Consequently, the hypothesis can be derived that the beetle also can open and close the cavities to expose or to uncouple the internal sensilla from the environment.

In addition to the features already mentioned in "General morphology of the antenna", cross sections through the cavities show that the inner lumina are round to oval (Fig. 7A-C). Thus, cavities can be regarded as laterally open tubes. Tube diameters slightly increase from the shorter inner part (about $12 \mu \mathrm{m}$ ) towards the posterior end of the longer outer part (up to $30 \mu \mathrm{m}$ ).
Toluidine-blue and Mallory staining revealed that the cuticle of a cavity and its surrounding consist of thin exocuticle only (Fig. 7A-C). At least the anterior part of the ventral bulge of the antennomers bearing the cavities is devoid of meso- and endocuticular layers (Fig. 7A). In Fig. 7A, B, it can be seen that the lips of the cavity stain much better than the surrounding exocuticle and, therefore, appear darker blue. This is indicative of softer cuticle. The presence of mesocuticle in this region is supported by the Mallory staining (Fig. 7C). Because the sensilla are tightly packed inside the cavity, the curved bottom is frequently perforated by the dendritic canals of the sensilla (Fig. 7A-C) resulting in an additional thinning of the cuticle. Because bases of most sensilla are equipped with a very thin socket (arrows in Fig. 7B, C; see also below), the cuticle of the cavities can easily be deformed. In case of a pressure increase inside the antennomer, the lips will open.

Many wax canals pass through the cuticle of the lips. Canals originate in wax glands located in the corner below the exocuticle and the adjoining cuticle of the cavity and run straight to the tip region of the lips (see arrow in Fig. 5B).

Three types of sensilla were found in the cavities:

(A) Olfactory sensilla basiconica. Multiporous singlewalled sensilla basiconica with a peg lengths of 4-5 $\mu \mathrm{m}$ represent the most common type of sensillum in the cavities (Fig. 8A, B). The cuticle surrounding the bases of the pegs is rather thin (Figs. 7B, C and 8A); however, we never found a mechanosensory innervation (i.e. a dendritic tip containing a tubular body ending at the base of the peg). Sensilla are innervated by the dendrites of two receptor cells (Fig. 8C, D). Both dendrites branch at the base of the peg into multiple thin terminal dendrites that continued to the top of the peg, most of them containing a single microtubulus. As a result, the lumen of the peg is densely filled with small dendrites (Fig. 8A, B).

In our sections, we always found the described conditions. However, the SEM investigation suggests that a few subtypes of the sensilla basiconica may be present with slightly differing peg lengths, forms and diameters.

(B) Sensilla coelocapitula. S. coelocapitula appear in the cavities in small numbers (Fig. 9). After opening up a cavity, small groups of sensilla were found mainly anteriorly (Fig. 9A, B). The mushroom-like pegs stick out of the surrounding cuticle a few hundred $\mathrm{nm}$ and are covered with microknobs (Fig. 9B, D, E). As a special feature, a crescent-shaped opening surrounds the pegs at one side (arrowheads in Fig. 9B). This narrow gap is the orifice of a small pit sunken in the cuticle (Figs. 7B and $9 \mathrm{C}, \mathrm{E})$. Thus, the small peg protrudes out of the pit on one side (Fig. 9C-E). Although fixation of the 


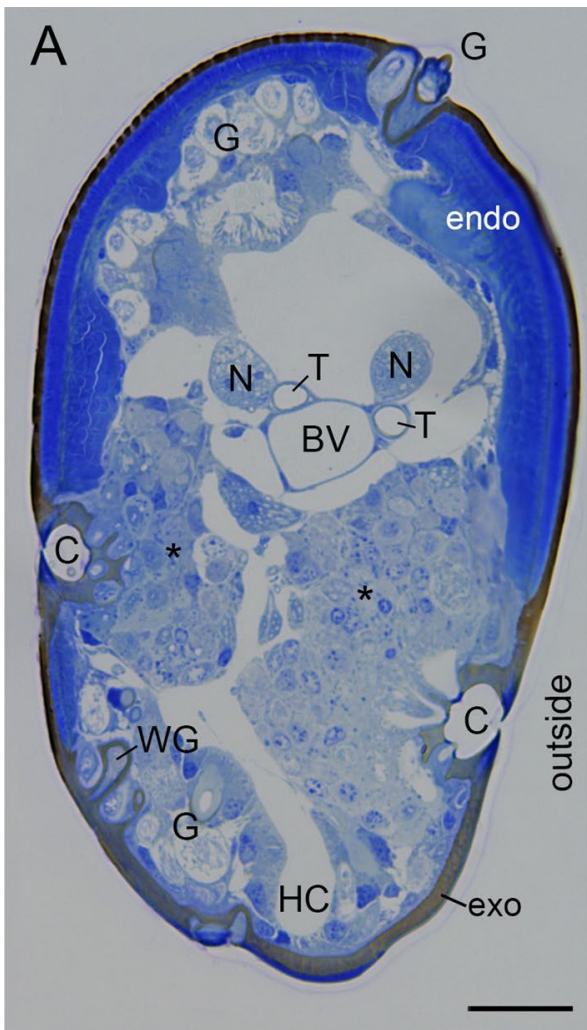

Fig. 7 Sensory cavities on antennomers 4-11. A Cross section through the anterior region of antennomer 7 showing the cavities (C). The cavity on the outside (on the right) is slightly larger and located more ventrally. Cavities are surrounded by massive clusters of receptor cells (asterisks). A central haemolymph lacuna (HC) extends ventrally ensuring nutrient supply. Haemolymph is pumped up to the outermost tip of the antenna through a blood vessel (BG). Dorsally and ventrally various sections through gustatory sensilla basiconica $(\mathrm{G})$ are visible. endo endocuticle, exo exocuticle, $N$ antennal nerves, $T$ trachea, $W G$ wax gland inside a cuticular tube; bar:

small sensilla was poor, it was found that at least two dendrites innervate the small peg (Figs. 7B and 9D, E). Dendrites are surrounded by a thick dendritic sheath that encloses the dendrites even at their outermost tips in the lumen of the peg (Fig. 9D inset, E). This is in line with the observation that no pores were found in the wall of the pegs ruling out any chemosensory function. A further peculiarity is the presence of electron-dense clots inside the outer receptor lymph cavity. Clots are often attached to the inner walls of the pits and the dendritic sheaths (Figs. 7B and 9C, D).

(C) Multiporous grooved peg sensilla (MGP). A modified type of sensillum basiconicum, called multiporous grooved peg sensillum (MGP), was also found in the cavities (Fig. 10). MGPs show a conical smooth basal part. In the distal half, about nine finger-like ribs and the corresponding number of grooves in between the ribs can be found. Like the sensilla coelocapitula,

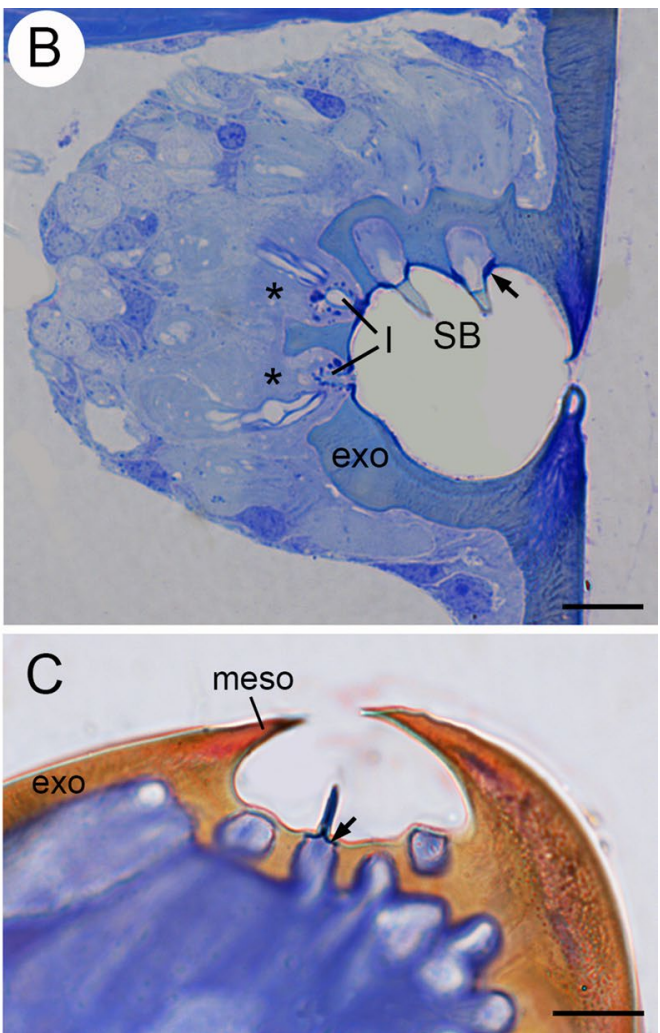

$25 \mu \mathrm{m}$. B Detail of an outer cavity and its surrounding sensory and enveloping cells. Inside the cavity two sensilla basiconica (SB) are visible. Two sections through sensilla semicoeloconica are apparent (asterisks). In each sensillum two dendrites are identifiable. Clods of dark blue stained material are located around the invaginations (I). Note the dark blue staining of the lips of the cavity (lower lip slightly deformed) indicating softer cuticle. Arrow indicates soft socket region; bar: $10 \mu \mathrm{m}$. C Mallory trichrome staining of the cuticle reveals reddish mesocuticle inside the lips of a cavity. Exocuticle appears amber; bar: $10 \mu \mathrm{m}$

MGPs occur in small groups of four to ten sensilla predominantly in the anterior region (Fig. 10A). The largest sensilla have a peg length of about $3 \mu \mathrm{m}$ and a basal diameter of 1.5-1.8 $\mu \mathrm{m}$. In dried specimens, pegs are embedded in a wrinkled base. However, this region does not represent a socket septum, but just a region of thinned cuticle (Fig. 10B, D). Furthermore, MGPs show a tendency to become shorter and some sensilla seem to be sunken into the cuticle. In these recessed sensilla, only the tip of the peg with the ribs is visible from outside (Fig. 10A).

Sections reveal that MGPs are double walled and innervated by four dendritic outer segments (Fig. 10C, D). Obviously, the dendrites do not branch in the double-walled pegs. The grooves between the ribs continue as pores connecting the outside to the inner lumen of the pegs with the dendrites 
Fig. 8 Sensilla basiconica inside the cavity. A Longitudinal section through a s. basiconicum. Multiple pores penetrate the thin cuticle of the peg. One of the two innervating dendrites is visible; branching of the terminal small subdendrites happens at the base of the peg (arrowhead); bar: $1 \mu \mathrm{m}$. B Cross section through the apical region of the peg. The lumen of the peg is densely filled with terminal dendrites dendrites; most of them containing one microtubulus. Numerous pores are visible as well as pore tubuli (arrowheads); bar: $0.5 \mu \mathrm{m}$. C Cross section through the two dendrites at the base of the peg (approximately at the level indicated by arrowhead in subfigure A). One dendrite has already started to branch in several subdendrites; the other remains unbranched; bar: $0.5 \mu \mathrm{m}$. D Cross section through the two unbranched dendritic outer segments (DOS) below the cuticle in the outer sensillum lymph space (OSLS): The dendritic sheath (DS) is somewhat swollen; bar: $0.5 \mu \mathrm{m}$
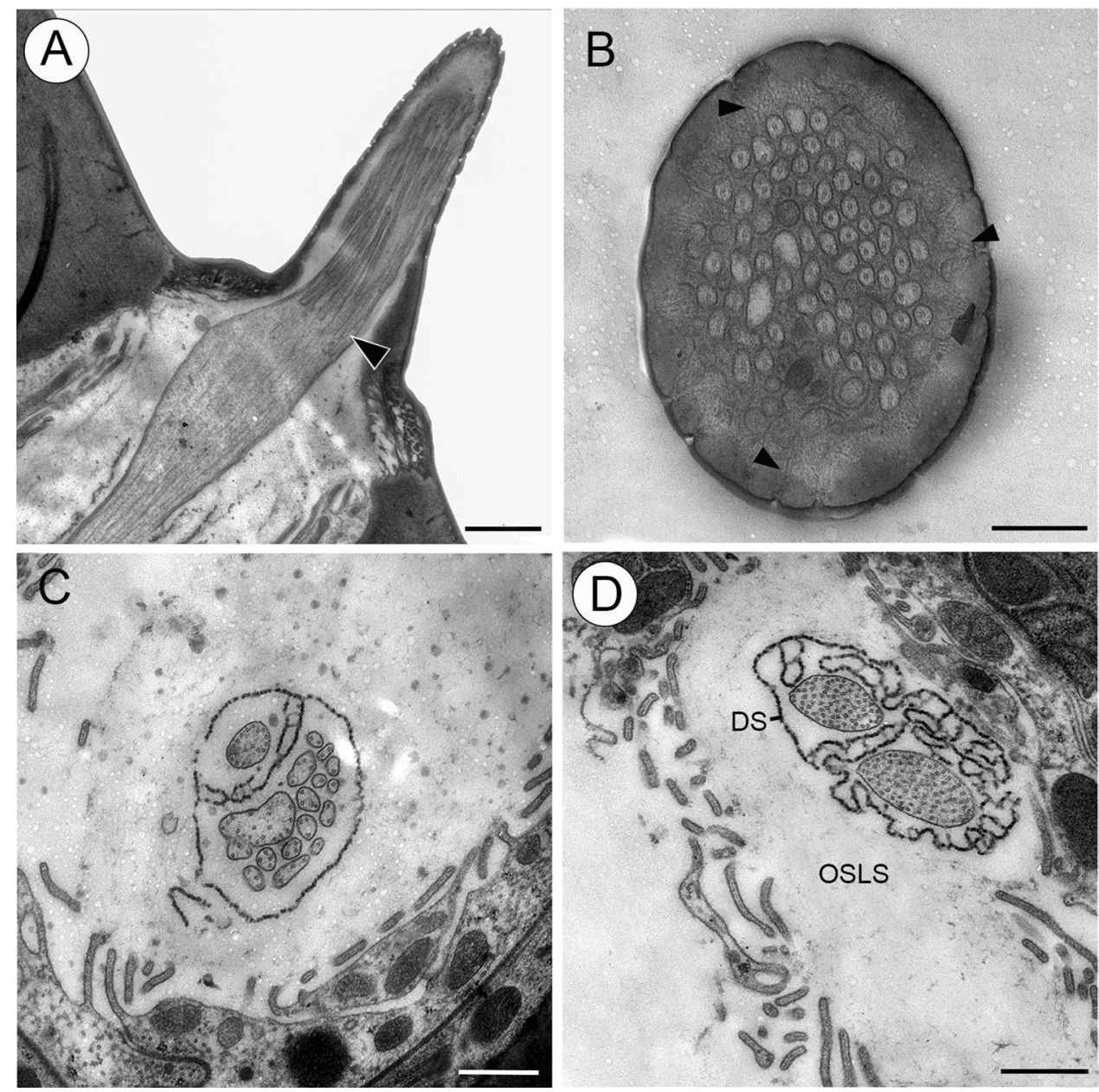

suggesting an olfactory function. Frequently, fascicular material was found in the pores (arrows in Fig. 10D).

\section{Discussion}

\section{Morphology and functional grouping of the antennal sensilla}

The distribution of the externally visible sensilla is clear and in principle corresponds to the description of the Merimna antenna published by Volkovitsh (Volkovitsh 2001). The glabrous dorsal and parts of the lateral surfaces are sparsely covered with aporous sensilla trichodea (e.g. hair mechanoreceptors), whereas the remaining lateral and ventral regions are covered with fields of short basiconic sensilla of two types: the first type (called U2 sensillum by Volkovitsh) is a uniporous contact chemoreceptor. These sensilla are frequently arranged in linear rows. This corresponds to the gustatory sensilla arranged in the fields $G_{\text {dorsal }}$ and $G_{\text {ventral }}$ described in our study (Fig. 1A). The second type (called B4c by Volkovitsh) is a multiporous olfactory sensillum.
This corresponds to the small ventral field of olfactory sensilla (O in Fig. 1B, C) of the present study. Because the cavities of the antennomers investigated by Volkovitsh were all closed, no information about the sensilla inside was given in the paper of Volkovitsh.

Consequently, the majority of the outer sensilla are contact mechano- and gustatory receptors. Only the small ventral field of very short multiporous basiconic sensilla most probably serves for olfaction. Therefore, it can be postulated that the external sensilla are mainly used for sensing on the ground. Numerous observations on freshly burnt areas have shown that the beetles intensively search for food (Schmitz et al. 2015). During this, they often inspect small cavities in the bark of burnt trees, obviously in search for scorched arthropods. During locomotor activity, the antennae are in permanent motion and are used for tactile and gustatory purposes. From this perspective, the morphology, dimensioning and arrangement of the mechano- and gustatory sensilla are reasonable. If the antenna is lowered and touches the ground, the ventrally located gustatory sensilla $\left(G_{\text {ventral }}\right.$ in Fig. 1B, C) will contact the substratum and provide gustatory information. Because the short pegs are inserted in a 

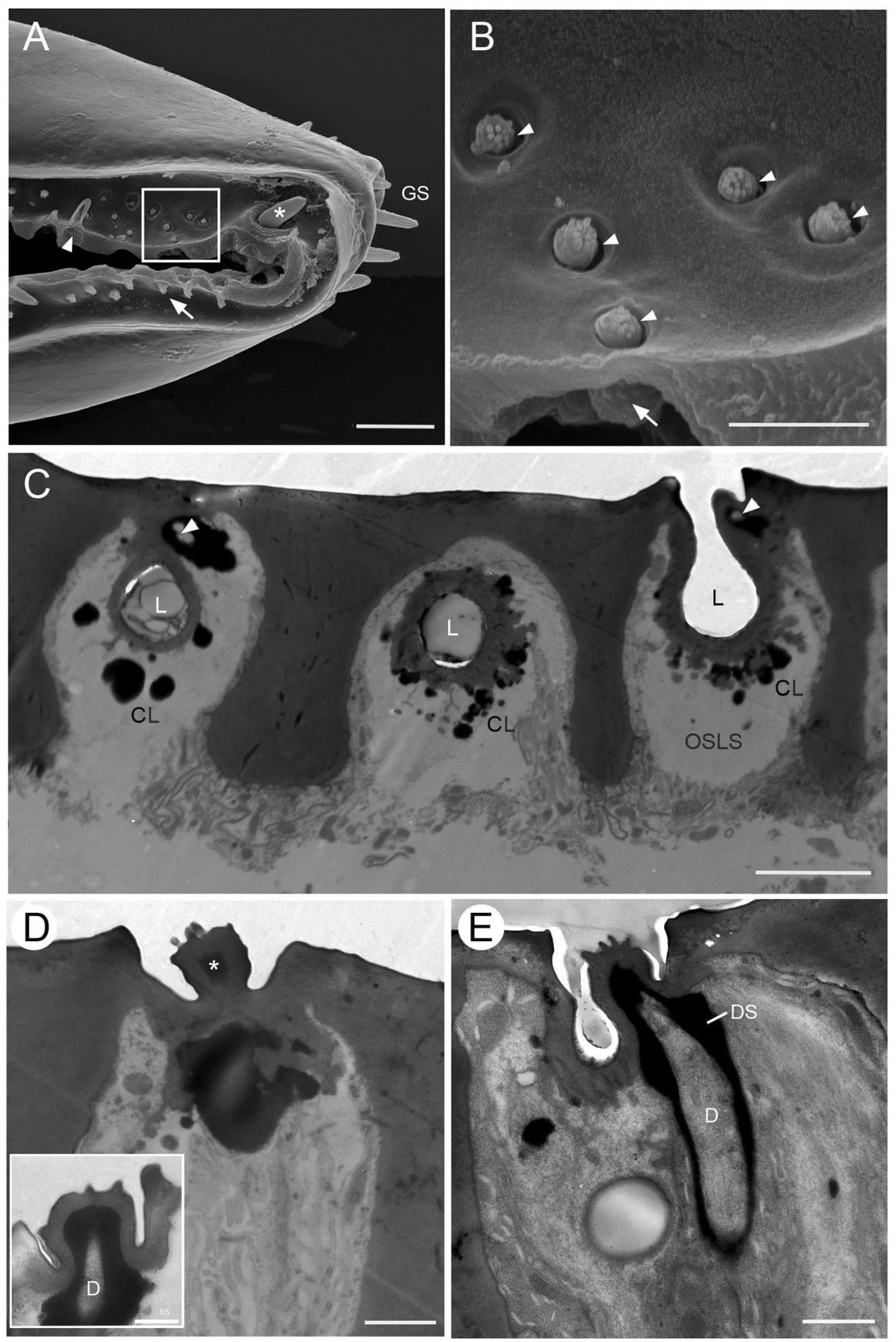

flexible socket and are innervated by a mechanoreceptor, tactile information is also obtained. The short mechanosensitive bristles interspersed between the gustatory sensilla are also involved in probing of surfaces with the gondola-shaped anterior parts of antennomers 4-11. If the antenna is lifted up and touches the wall of a hole above the beetle, the dorsal row of gustatory sensilla $\left(\mathrm{G}_{\text {dorsal }}\right.$ in Fig. 1B) will come in contact with the surface and gain gustatory and tactile information in the same way. The longer mechanosensitive bristles on the dorsal and lateral surfaces will provide additional mechanosensitive information. Because probing of the ground and potential food can be very intense, the length of the mechanosensitive bristles may have been reduced during the course of evolution in an anterior-ventral direction to ensure that bristles of the ventral region do not break off. Theoretically, the gustatory sensilla could also be used for 
4Fig. 9 Sensilla coelocapitula. A View into the dorsal half of the anterior cavity on distal antennomer 11; ventral half removed. In the shorter inner cavity (below) seven grooved peg sensilla (GPS) are located (arrow). In the above outer cavity, a sensillum basiconicum (arrowhead) and a field of 9 sensilla coelocapitula (5 enclosed by white rectangle) can be seen. Asterisk marks a larger gustatory sensillum also included in the cavity. GS: gustatory sensilla on top of the antennomer; bar: $15 \mu \mathrm{m}$. B Small field of five sensilla coelocapitula encircled by the white rectangle in subfigure A. Arrowheads mark crescent-shaped openings of the pits below the cuticle. Arrow points to the wall of the pit of the lower sensillum; bar: $4 \mu \mathrm{m}$. C Cuts through three sensilla coelocapitula at different planes. In all sensilla the lumina (L) of the pits are visible The dark clots (CL) are situated in the outer sensillum lymph space (OSLS) often attached to the walls of the pits. In the left and right sensillum the distal tip of a dendrite embedded in electron-dense material can be discerned (arrowheads). The outermost parts of the pegs are out of plane of the section; bar: $3 \mu \mathrm{m}$. D Peripheral cut through a sensillum including the peg. The invagination is out of plane. Note electron-dense material inside the peg lumen (asterisk) and below the peg; bar: $1 \mu \mathrm{m}$. Inset: cut through center of a peg filled with electron-dense material, $\mathbf{D}$ dendrite; bar: $0.5 \mu \mathrm{m}$. E Cut through a sensillum showing the innervation of the peg by a dendrite (D). The massive dendritic sheath (DS) around the dendrite extends into the nubby peg. The invagination of the edge of the pit is visible; bar: $1 \mu \mathrm{m}$

olfactory purposes as it has been proposed for some simuliid flies and orb-weaving spiders (McIver et al. 1980; Müller et al. 2020). Future electrophysiological experiments have to reveal whether this assumption is correct.

The field of olfactory sensilla located on the posterior ventral part of the gondolas (antennomeres 4-11) leads over to the use of the antenna in flight. Currently, it is not clear if this small group of olfactory receptors is mainly used on the ground or in flight. As an exceptional feature, it was found that the numbers and diameters of dendrites inside the multiporous peg are very small compared to the situation in the olfactory sensilla basiconica in the cavities (see below). This may indicate a relatively low sensitivity. Thus, it can be speculated that sensilla are used on the ground by a foraging beetle close to a possible source of food.

On the other hand, it can be proposed that the sensilla inside the cavities are used in flight for detecting and approaching a fire by olfactory cues. The majority of cavity sensilla are multiporous thin-walled basiconic sensilla. The pegs are densely filled with highly branched dendrites that are at risk of drying out when exposed to high temperatures and low humidity. Both are predominant on a freshly burnt area on which Merimna can be found for a few days. Thus it is postulated that the cavities are closed when beetles stay on a postfire area. However, in flight the cavities most probably will be open, allowing an intake of air. The airflow will enter the tubular lumen of the cavity through the inner/ anterior part of the slit (see arrow in Fig. 1C). Because the sensilla inside the cavities are tightly adjoined, flow of air around the numerous pegs will be turbulent and, therefore, will be slowed down. This may result in a concentration effect of odour molecules inside the cavities. Consequently, an intensive contact of odourants with the peg surfaces can be assumed until the air leaves the cavity along the longer slit on the outside. More information about the three types of sensilla found in the cavities will be given in the next chapters.

\section{Fire adaptions}

Merimna can be classified as a highly thermo- and pyrophilous insect (Hawkeswood and Peterson 1982; Schmitz and Schmitz 2002; Schmitz et al. 2015). Attracted by fires in Eucalyptus forests, beetles of both sexes start reproduction and search for food on the freshly burnt area. Beetles are around as long as the postfire area is "active", which means that remnants of burning or glowing wood or spots of hot ashes emit smoke and radiant heat. Therefore, the area temporary populated by Merimna is a hazardous environment not only for insects. Due to the fire-related defoliation of trees and shrubs, most of the solar radiation reaches the ground causing high surface and air temperatures often beyond $35{ }^{\circ} \mathrm{C}$. As if that was not enough, Merimna shows intense basking behaviour by resting on the black bark of sunlit burnt trees. As a consequence, body temperatures may rise up to $45^{\circ} \mathrm{C}$ (Schmitz et al. 2015). In search for food, beetles often can be observed running over the bark, covered with ash, or the ground resulting in heavy contamination of the entire body surface.

As a result, especially the antennal sensilla with pores and thin flexible sockets should be protected from high temperatures, desiccation and pollution. A first preadaptation in that regard is the low number of external antennal sensilla already present in all members of the tribus Melanophilini including the pyrophilous genera Merimna and Melanophila as well as the non-pyrophilous genera Juniperella, Xenomelanophila, and Phaenops (Volkovitsh 2001). Most probably, the thermophilic biology of this group has resulted in a reduction of the numbers of delicate external sensilla. Obviously, this tendency is even more enhanced in Merimna. A further specific fire adaptation can be revealed by comparing the lengths of antennal sensilla between the non-pyrophilous Melanophilini and Merimna (images of antennomeres provided, e. g. in Volkovitsh 2001). The considerable reduction of the lengths of nearly all external sensilla in Merimna is striking. This is particularly true for the ventral olfactory sensilla with many pores in the pegs (Figs. 1B, C and 2A). An even more efficient step in adapting the antennae to a hot and dirty environment is the enclosure of desiccation-sensitive sensilla in a protective internal cavity. This mechanism can already be found to varying degrees in many other non-pyrophilous Buprestidae. It starts with the occurrence of well delineated sensory pits or fossae 
Fig. 10 Multiporous grooved peg sensillum (MGP). A SEM micrograph of 4 MGP. One sensillum nearly fully hidden in a pit; bar: $1 \mu \mathrm{m}$. B Oblique section through a sensillum showing sections of two fingerlike ribs and the central dendrites (D) enclosed in the dendritic sheath (DS); bar: $1 \mu \mathrm{m}$. C Cross section through the cuticular canal below the peg. The outer sensillum lymph space (OSLS) is visible containing outer dendritic segments (DOS) of 4 receptor cells enclosed in the dendritic sheath (DS); TRI trichogen cell; $T O$ tormogen cell; bar: $1 \mu \mathrm{m}$. D Oblique section through a sensillum revealing the lacunae in the fingerlike ribs and, thereby, the double-walled structure of the sensillum.

Arrows indicate pores between the ribs obviously filled with a fascicular content; $D$ dendrite in the central lumen; bar: $0.5 \mu \mathrm{m}$. Inset: cross section through the fingerlike bulbous head showing pores filled with fascicular material (arrows); bar: $0.25 \mu \mathrm{m}$
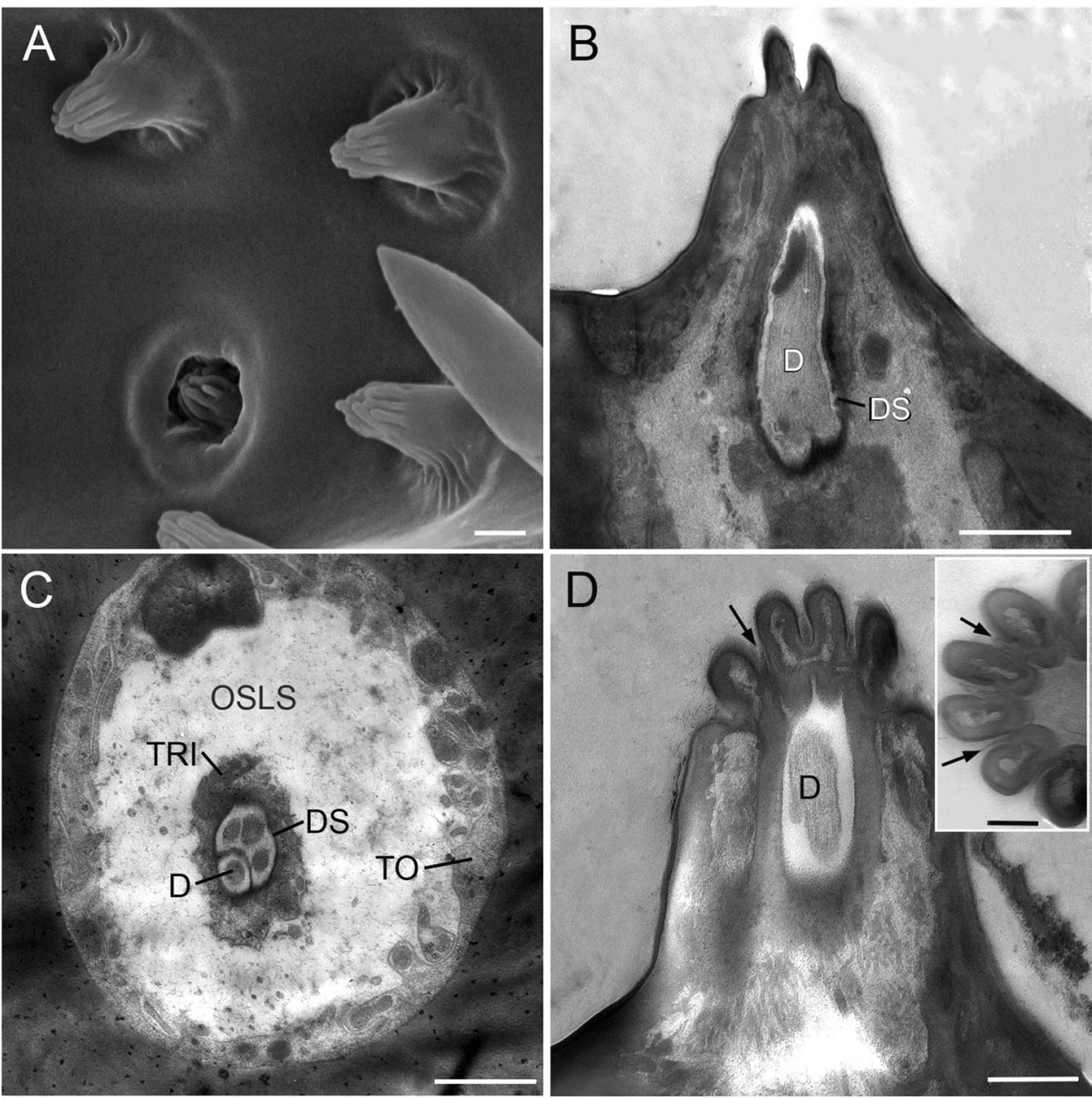

mainly located on the anterior regions of the antennomers. Finally, sensory cavities are developed that are partially or totally invaginated into the cuticle. From outside, mostly only a narrow slit can be discerned (Volkovitsh 2001). However, not many details about morphology and mechanics of fully closable cavities in jewel beetles are known. Our results show that the efficiency with which the cavity sensilla can be isolated from the surrounding seems to be high. When the cavities are closed, the soft sealing lips of the cleft perfectly fit together resulting in a tight seal. Additionally, the wax released by the canals running from the wax glands to the tips of the lips (Fig. 5B) most probably further improves the seal. As a result, the sensilla inside the cavities are fully protected against desiccation and pollution. Therefore, it is proposed that cavities are open in flying beetles searching for a fire by olfaction. It has been shown that a part of the antennal sensilla of Merimna are highly sensitive to eucalyptol and furfural released by burning eucalyptus wood. A couple of other volatile organic compounds (VOCs) characteristic for burning wood can also be perceived (Paczkowski 2018).
Inside the cavities the olfactory basiconic and multiporous grooved peg sensilla (MGPs) are good candidates for the detection of fire-specific VOCs. As described by Volkovitsh 2001 for several other genera of Buprestidae (e. g. Mendizabalia, Maoraxia, Galbella), MGPs occur singly or in small groups and are usually incorporated into apical organs. More recently, MGPs have also been found in the buprestid beetles Lamprodila festiva (Faucheux et al. 2020) and Agrilus mali (Yi et al. 2016). Based on their morphology as double-walled sensilla with spoke channels, a function in olfaction is likely (Steinbrecht 1997).

Opening and closing of the cavities may be achieved by increasing or decreasing the haemolymph pressure inside the antennae as it has been shown in the cockchafer Melolontha melolontha. The cockchafer has an elaborated antennal circulatory system allowing an increase in intraantennal pressure that most probably results in spreading the lamellate antennal club (Pass 1980). Last not least, the general abundance of wax pores in the cuticle also points towards a consistent outer wax layer on the surface of the antenna reducing transcuticular evaporation. 


\section{Thermoreceptors}

One main objective of the current study was the identification of thermo-/hygroreceptors on the antenna of Merimna. However, those receptors are not easy to identify because the sensilla housing thermo-/hygroreceptors can show quite different shapes. Thermo-/hygroreceptive receptor cells have been found in small aporous pegs sunken to varying extents into tubular cavities or pits, in multiporous double-walled sensilla, and also in tapering hairs (Altner et al. 1983; Altner and Loftus 1985; Tichy and Loftus 1996; Zopf et al. 2014; Nagel and Kleineidam 2015). The most common type found in many different insect orders is a thermo-/hygroreceptive sensillum coeloconicum, also called peg-in-pit sensillum, where a short aporous peg inside a narrow pit is innervated by a triad of receptor cells: two hygroreceptors working antagonistically (a moist and a dry cell) and one thermoreceptor (often a cold cell with a lamellated dendrite) (Altner and Loftus 1985). However, such a typical s. coeloconicum was not found in our study.

Therefore, a look onto thermo-/hygroreceptors known in beetles and especially in Buprestidae seems promising. Up to now, two reports deal with possible thermo-/hygroreceptors in jewel beetles. The first one is a more general note made by Volkovitsh in his comprehensive work about the antennal sensilla in Buprestidae (Volkovitsh 2001). The author mentioned that thermoreceptors may exist in sensilla termed B4 (a single-walled multiporous basiconic sensillum). However, no further proof is provided for this statement. Additionally, up to now thermoreceptors have not been determined in multiporous single-walled sensilla (Nagel and Kleineidam 2015). The second hint is given in the study of Scott and Gara 1975 (cf. Introduction). Here, an SEM image of a putative sensillum campaniformium is shown that was found on several antennomers in females of the pyrophilous jewel beetle Melanophila acuminata, a close relative of Merimna. The authors claimed that this campaniform sensillum may act as a thermoreceptor without giving further evidence. Interestingly, more than 25 years later, another publication appeared stating that campaniform sensilla work as a thermoreceptors in beetles (Merivee et al. 2003). Sensilla can be found on the anterior antennomeres in several carabid and elaterid beetles (Merivee et al. 1998, 2001) and it turned out that the sensilla are innervated by a typical triad of hygro- and thermoreceptors mentioned above (Merivee et al. 2010). Therefore, in their later work, the authors renamed this type of sensillum into dome-shaped sensillum (DSS) and provided convincing electrophysiological evidence for a thermo-/hygroreceptive function (Merivee et al. 2010, 2020; Nurme et al. 2015). According to the considerations published by Nagel and Kleineidam (Nagel and Kleineidam 2015), this thermo-/ hygroreceptive sensillum can be regarded as a beetle-specific sensillum coelocapitulum. In a sensillum coelocapitulum, a tiny mushroom-like protrusion is situated in the centre of a shallow pit (Esslen and Kaissling 1976; Yokohari 1983; Tichy and Kallina 2014; Nagel and Kleineidam 2015). In the beetle-specific DSS, this depression obviously has bulged out now forming a small dome. Importantly, the sensory inventory is the same as in a sensillum coelocapitulum.

In the absence of a more reasonable alternative we also have classified the smallest cavity sensillum as sensillum coelocapitulum (Fig. 9). It shows a tiny mushroom-like protrusion in a shallow depression (Fig. 9B). However, there are differences to the sensilla coelocapitula described previously. One striking difference is that the small peg arises laterally as part of the wall of a larger pit and protrudes a few hundred $\mathrm{nm}$ above the edge of the narrow pit opening. Therefore, this sensillum seems to be intermediate between a peg-in-pit sensillum (sensillum coeloconicum) and a sensillum coelocapitulum. From an evolutionary point of view, it can be speculated that the peg is "on the way" from the bottom of a former pit towards a more exposed position on the surface. The sheltered position inside the cavity could have made the protection of a peg at the bottom of a pit expendable. To increase the exposition of the peg without the risk of damage, a process of evolution could have started to relocated the peg to the outside. However, this is a pure guess. Which function the pit may have is still an open question.

Another difference is the number of sensory dendrites innervating the peg. We found no typical triad of sensory dendrites, but only two unbranched dendritic outer segments linked with their outermost tips to the peg by their massive dendritic sheaths. Due to the moderate fixation we could not obtain more information about the ultrastructure of the dendrites. So it may be possible that the dendrites belong to two hygroreceptors. However, sensilla coeloconica or s. coelocapitula innervated by only two hygroreceptors have not been described so far (Tichy and Loftus 1996; Tichy et al. 2017; Enjin 2017). Therefore, we postulate that the sensillum coelocapitulum of Merimna is innervated by two antagonistically acting thermoreceptors as described for sensilla coeloconica in some other insects (Davis and Sokolove 1975; McIver and Siemicki 1985; Zopf et al. 2014; Schneider et al. 2018).

A prominent trait of the sensilla coelocapitula is the presence of electron-dense material. Firstly, massively thickened dendritic sheaths (ds) surround the entire dendrites, especially at their tips. As a consequence, the lumen of the peg is mainly filled out by the dark ds material. Secondly, several irregular dark clots inside the outer receptor lymph cavity were observed that are frequently attached to the wall of the pit. The origin of this material is unclear. It can be speculated that it consists of the same material as the ds and, therefore, has been sequestered by the thecogen cell. Electron-dense material and especially a thick ds seems to be characteristic for many thermo-/hygroreceptors (Altner et al. 
1981, 1983; Yokohari 1983; Kreiss et al. 2005; Schneider et al. 2018).

One possible role of the dense material could be heat conduction. In the so-called disc sensilla of the pyrophilous `Little Ash Beetle`Acanthocnemus nigricans, rods of electron-dense material were found (Kreiss et al. 2005). In Acanthocnemus, a pair of prothoracic discs serve as IR receptors and are covered with about 90 microsensilla (Kreiss et al. 2007). The rods connect the small outer pegs of a sensillum to the short dendritic inner segments of the receptor cells inside a disc. A rod most probably consist of the hypertrophied ds that have totally displaced the dendritic outer segment of the thermoreceptive receptor cell. Conduction of heat generated in the outer peg and its surrounding may be conducted by the rods to the thermoreceptive receptor cell (Zhou et al. 2016). A similar function has also been discussed for the thick dendritic sheaths of the thermoreceptors in a tropical katydid of the genus Mecopoda (Schneider et al. 2018).

Another function may be infrared (IR) absorption as most organic molecules including the arthropod cuticle strongly absorb IR between 3 and $4 \mu \mathrm{m}$ in the mid-wavelength IR and between 8 and $10 \mu \mathrm{m}$ in the long-wavelength IR (Hesse et al. 1995; Schmitz et al. 2016). Therefore, a local increase of density of an IR absorbing biopolymer can considerably increase the absorption probability of IR photons (Schmitz et al. 2016). However, that the sensilla coelocapitula in the cavities on the Merimna antenna function as IR receptors seems unlikely because of their hidden position. On the other hand, if the cavities are opened, IR radiation may enter the sensilla thus allowing additional perception of IR radiation.

As long as electrophysiological data are not available, the question regarding the possible thermo-/hygroreceptive function of the sensilla coelocapitula on the Merimna antenna remains open. However, according to our results, this type of sensillum is the only one on the Merimna antenna that could have a thermo-/hygroreceptive function.

\section{Concluding remarks}

After arrival on a freshly burnt area Merimna is exposed to a thermally dangerous surroundings. In line with it, we found a couple of morphological adaptations of the antennae to protect the sensilla against desiccation and pollution. However, we found no evidence for an increased presence of antennal thermo-/hygroreceptors. Former SEM investigation revealed that there is strong evidence for the existence of s. coeloconica/s. coelocapitula-like sensilla in other jewel beetles (Volkovitsh 2001; Faucheux et al. 2020). These tiny sensilla are mainly incorporated into apical depressions and cavities and could serve as thermo-/hygroreceptors as proposed in our study for Merimna. Although we opened up the antennomers to ensure the fixatives a good access to the tissue, especially the preservation of the sensilla coelocapitula was only moderate. Thus, future ultrastructural investigations using cryofixation or high pressure freezing have to yield more detailed information about the ultrastructure of the receptor cells innervating sensilla coelocapitula in jewel beetles.

It can be assumed that Merimna mainly relies on its abdominal IR organs not to come to harm when sojourning on a "hot" postfire area (Schmitz et al. 2015; Hinz et al. 2018).

Acknowledgements We are indebted to the Department of Biodiversity, Conservation and Attractions (DBCA) in Western Australia for issuing the Fauna Taking Licences No. FO25000232 from 06/01/2020. Brian Inglis, Clayton Sanders and Leigh Sage from the Parks and Wildlife Service of DBCA in Wanneroo permitted access to the burnt areas and supported our fieldwork. John Angus from the DBCA Science Division provided lab space in the Woodvale Research Centre in Woodvale, WA. We would like to thank the people from the Nees Institute of the University of Bonn for allowing us to use their SEM. We thank two anonymous referees for their helpful comments.

Author contributions All authors contributed to the study conception and design. Material preparation, data collection and analysis were performed by AS and HS. The first draft of the manuscript was written by HS and all authors commented on previous versions of the manuscript. All authors read and approved the final manuscript.

Funding Open Access funding enabled and organized by Projekt DEAL. Supported by grant SCHM 1161/16-1 from the German Research Foundation (DFG) to HS.

Availability of data and material Data sharing is not applicable to this article as no datasets were generated or analysed during the current study.

Code availability Not applicable.

\section{Declarations}

Conflict of interest The authors declare that they have no conflict of interest.

Ethics approval No approval of research ethics committees was required to accomplish the goals of this study because experimental work was conducted with an unregulated invertebrate species.

Consent to participate Not applicable.

Consent for publication Not applicable.

Open Access This article is licensed under a Creative Commons Attribution 4.0 International License, which permits use, sharing, adaptation, distribution and reproduction in any medium or format, as long as you give appropriate credit to the original author(s) and the source, provide a link to the Creative Commons licence, and indicate if changes were made. The images or other third party material in this article are included in the article's Creative Commons licence, unless indicated otherwise in a credit line to the material. If material is not included in 
the article's Creative Commons licence and your intended use is not permitted by statutory regulation or exceeds the permitted use, you will need to obtain permission directly from the copyright holder. To view a copy of this licence, visit http://creativecommons.org/licenses/by/4.0/.

\section{References}

Altner H, Loftus R (1985) Ultrastructure and function of insect thermoand hygroreceptors. Ann Rev Entomol 30:273-295

Altner H, Routil C, Loftus R (1981) The structure of bimodal chemo-, thermo-, and hygroreceptive sensilla on the antenna of Locusta migratoria. Cell Tissue Res 215:289-308

Altner H, Schaller-Selzer L, Stetter H, Wohlrab I (1983) Poreless sensilla with inflexible sockets. Cell Tissue Res 234:279-307

Bari G, Scala A, Garzone V, Salvia R, Yalcin C, Vernile P, Aresta AM, Facini O, Baraldi R, Bufo SA (2019) Chemical ecology of Capnodis tenebrionis (L.) (Coleoptera: Buprestidae): behavioral and biochemical strategies for intraspecific and host interactions. Front Physiol 10:604

Bellamy CL (2002) Zoological catalogue of Australia volume 29.5 Coleoptera: Buprestoidea, vol 29.5. CSIRO Publishing, Melbourne

Crook D, Kerr L, Mastro V (2008) Distribution and fine structure of antennal sensilla in emerald ash borer (Coleoptera: Buprestidae). Ann Entomol Soc Am 101(6):1103-1111

Davis EE, Sokolove PG (1975) Temperature response of antennal receptors of the mosquito, Aedes aegypti. J Comp Physiol 96:223-236

Enjin A (2017) Humidity sensing in insects-from ecology to neural processing. Curr Opin Insect Sci 24:1-6

Esslen J, Kaissling KE (1976) Zahl und Verteilung antennaler Sensillen bei der Honigbiene (Apis mellifera). Zoomorphologie $83: 227-251$

Faucheux MJ, Németh T, Hoffmannova J, Kundrata R (2020) Scanning electron microscopy reveals the antennal micromorphology of Lamprodila (Palmar) festiva (Coleoptera: Buprestidae), an invasive pest of ornamental Cupressaceae in Western Palaearctic. Biology 9(11):375

Gaffal KP (1979) An ultrastructural study of the tips of four classical bimodal sensilla with one mechanosensitive and several chemosensitive receptor cells. Zoomorphologie 92(3):273-291

Hawkeswood TJ (2007) Review of the biology of the genus Merimna Saunders, 1868 (Coleoptera: Buprestidae). Calodema 9:12-13

Hawkeswood TJ, Peterson M (1982) A review of the larval host records for Australian jewel beetles (Coleoptera: Buprestidae). Victorian Naturalist 99:240-251

Hesse M, Meier H, Zeeh B (1995) Spektroskopische Methoden in der orgnischen Chemie, 5th edn. Georg Thieme Verlag Stuttgart

Hinz M, Klein A, Schmitz A, Schmitz H (2018) The impact of infrared radiation in flight control in the Australian "firebeetle" Merimna atrata. PLoS One 13(2):e0192865

Kreiss EJ, Schmitz A, Schmitz H (2005) Morphology of the prothoracic discs and associated sensilla of Acanthocnemus nigricans (Coleoptera, Acanthocnemidae). Arthropod Struct Dev 34(4):419-428. https://doi.org/10.1016/j.asd.2005.06.001

Kreiss E-J, Schmitz H, Gebhardt M (2007) Electrophysiological characterisation of the infrared organ of the Australian "Littel Ash Beetle" Acanthocnemus nigricans (Coleoptera, Acanthocnemidae). J Comp Physiol A 193:729-739

McIver S, Siemicki R (1985) Fine structure of antennal putative thermo-/hygrosensilla of adult Rhodnius prolixus Stål (Hemiptera: Reduviidae). J Morphol 183(1):15-23
McIver S, Siemicki R, Sutcliffe J (1980) Bifurcate sensilla on the tarsi of female black flies, Simulium venustum (Diptera: Simuliidae): contact chemosensilla adapted for olfaction? J Morphol 165(1):1-11

Merivee E, Rahi M, Bresciani J, Ravn HP, Luik A (1998) Antennal sensilla of the click beetle, Limonius aeruginosus (Olivier) (Coleoptera: Elateridae). Int J Insect Morphol Embryol 27(4):311-318

Merivee E, Ploomi A, Luik A, Rahi M, Sammelselg V (2001) Antennal sensilla of the ground beetle Platynus dorsalis (Pontoppidan, 1763) (Coleoptera, carabidae). Microsc Res Tech 55(5):339-349

Merivee E, Vanatoa A, Luik A, Rahi M, Sammelselg V, Ploomi A (2003) Electrophysiological identification of cold receptors on the antennae of the ground beetle Pterostichus aethiops. Physiol Entomol 28(2):88-96

Merivee E, Must A, Luik A, Williams I (2010) Electrophysiological identification of hygroreceptor neurons from the antennal domeshaped sensilla in the ground beetle Pterostichus oblongopunctatus. J Insect Physiol 56(11):1671-1678

Merivee E, Must A, Nurme K, Di Giulio A, Muzzi M, Williams I, Mänd M (2020) Neural code for ambient heat detection in elaterid beetles. Front Behav Neurosci 14:1

Müller CH, Ganske AS, Uhl G (2020) Ultrastructure of chemosensory tarsal tip-pore sensilla of Argiope spp. Audouin, 1826 (Chelicerata: Araneae: Araneidae). J Morphol 281(12):1634-1659

Nagel M, Kleineidam CJ (2015) Two cold-sensitive neurons within one sensillum code for different parameters of the thermal environment in the ant Camponotus rufipes. Front Behav Neurosci 9:240

Noirot C, Quennedey A (1991) Glands, gland cells, glandular units: some comments on terminology and classification. Annales De La Société Entomologique De France 2:123-128

Nurme K, Merivee E, Must A, Sibul I, Muzzi M, Di Giulio A, Williams I, Tooming E (2015) Responses of the antennal bimodal hygroreceptor neurons to innocuous and noxious high temperatures in the carabid beetle, Pterostichus oblongopunctatus. J Insect Physiol 81:1-13

Paczkowski S (2018) Wood fire detection by imitating beetle olfaction with gas sensors. Curr Trends Entomol. https://doi.org/10.29011/ CTEZS-105.000005

Pass G (1980) The anatomy and ultrastructure of the antennal circulatory organs in the cockchafer beetle Melolonth melolontha $\mathrm{L}$. (Coleoptera, Scarabaeidae). Zoomorphology 96(1-2):77-89

Poulton EB (1915) The habits of the Australian buprestid "fire-beetle" Merimna atrata, Lap. et Gory. In: Transactions of the Entomological Society of London, Pt 1, Proceedings, pp 3-4

Schmitz H, Schmitz A (2002) Australian fire-beetles. Landscope Spring 2002:36-41

Schmitz A, Schmitz H (2020) Unusual discovery of the 'Australian Firebeetle' Merimna atrata on an older postfire area. Rec West Aust Mus 50(052):052

Schmitz H, Mürtz M, Bleckmann H (2000) Responses of the infrared sensilla of Melanophila acuminata (Coleoptera : Buprestidae) to monochromatic infrared stimulation. J Comp Physiol A 186(6):543-549

Schmitz A, Schneider ES, Schmitz H (2015) Behaviour of the Australian "fire-beetle" Merimna atrata (Coleoptera: Buprestidae) on burnt areas after bushfires. Rec West Aust Mus 30(1):1-11

Schmitz H, Schmitz A, Schneider E (2016) Matched filter properties of infrared receptors used for fire and heat detection in insects. In: von der Emde G, Warrant E (eds) The ecology of animal senses. Springer International Publishing, pp 207-234. https://doi.org/10. 1007/978-3-319-25492-0_8

Schneider ES, Kleineidam CJ, Leitinger G, Römer H (2018) Ultrastructure and electrophysiology of thermosensitive sensilla coeloconica in a tropical katydid of the genus Mecopoda (Orthoptera, Tettigoniidae). Arthropod Struct Dev 47(5):482-497 
Scott DW, Gara RJ (1975) Antennal sensory organs of two Melanophila species (Coleoptera: Buprestidae). Ann Entomol Soc Am 68(5):842-846

Steinbrecht RA (1997) Pore structures in insect olfactory sensilla: a review of data and concepts. Int J Insect Morphol Embryol 26(3-4):229-245

Tepper JGO (1887) Common native insects of South Australia: a popular guide to South Australian entomology. Part 1. Coleoptera or Beetles. E.S. Wigg \& Son, Adelaide, pp 1-46

Tichy H, Loftus R (1996) Hygroreceptors in insects and a spider: humidity transduction models. Naturwissenschaften 83:255-263

Tichy H, Hellwig M, Kallina W (2017) Revisiting theories of humidity transduction: a focus on electrophysiological data. Front Physiol 8:650

Tichy H, Kallina W (2014) Sensitivity of honeybee hygroreceptors to slow humidity changes and temporal humidity variation detected in high resolution by mobile measurements. PLoS One 9(6): e99032

Volkovitsh M (2001) The comparative morphology of antennal structures in Buprestidae (Coleoptera): evolutionary trends, taxonomic and phylogenic implications. Part 1. Acta Musei Moraviae. Scientia Biologicae (brno) 86:43-169

Weyda F (1982) Adaptation of Mallory's trichrome stain to insect tissue epoxy sections. Zeitschrift Für Mikroskopsich-Anatomische Forschung 96:79-80
Yi Z, Liu D, Cui X, Shang Z (2016) Morphology and ultrastructure of antennal sensilla in male and female Agrilus mali (Coleoptera: Buprestidae). J Insect Sci 16(1):87

Yokohari F (1983) The coelocapitular sensillum, an antennal hygro-and thermoreceptive sensillum of the honey bee, Apis mellifera L. Cell Tissue Res 233(2):355-365

Zhou Z, Gong Y, Yang D, Schmitz A, Schmitz H (2016) Function modeling of the infrared organ of "Little ash beetle" Acanthocnemus Nigricans (Coleoptera, Acanthocnemidae). J Bionic Eng 13(4):650-658. https://doi.org/10.1016/S1672-6529(16)60336-0

Zopf LM, Lazzari CR, Tichy H (2014) Differential effects of ambient temperature on warm cell responses to infrared radiation in the bloodsucking bug Rhodnius prolixus. J Neurophysiol 111(6):1341-1349

Publisher's Note Springer Nature remains neutral with regard to jurisdictional claims in published maps and institutional affiliations. 